\title{
DOES A 'TWO-STREAM' FLOW MODEL APPLY TO WAKES OF LARGE BODIES IN SPACE?
}

\author{
(Letter to the Editor)
}

\author{
URI SAMIR \\ Space Physics Research Laboratory, Univ. of Michigan, Mich., U.S.A. \\ and \\ Dept. of Environmental Sciences, Tel-Aviv Univ., Israel \\ and \\ N. H. STONE and W. A. ORAN \\ Space Sciences Laboratory, Marshall Space Flight Center, \\ Huntsville, Ala., U.S.A.
}

(Received 3 July, 1974)

\begin{abstract}
Structural patterns of ion and electron currents observed on the wake axes of the Ariel I and the Gemini 10 space vehicles are re-examined, together with relevant theoretical and laboratory 'simulation' studies. Some insight into existing in-situ data is provided. The possibility that 'converging-stream' models describe structural features of current enhancements in the wake region of large spacecraft is discussed.
\end{abstract}

The only available axial profiles of charged particle currents in the wake of a large spacecraft were presented by Troy et al. (1970), based on the wake experiment of the Gemini/Agena 10 spacecraft system. The probes mounted on the Agena vehicle measured both the ion and electron currents in the wake created by the Gemini as the two vehicles moved apart after an undocking manceuver. These data (which consisted of a single profile each for ion and electron currents) are shown in Figure 1. The spacecraft configuration is shown in Figure 2a. At about $400 \mathrm{~km}$ altitude, where the above profiles were measured, the ratio $\left(R_{\mathrm{G}} / \lambda_{\mathrm{D}}\right)$ is of the order of 100 , where $R_{\mathrm{G}}$ is the 'effective' body radius for the Gemini and $\lambda_{\mathrm{D}}$ is the Debye length. The irregularity in $\left(I_{\mathrm{c}} / I_{\mathrm{e} 0}\right)$ at $\left(r / R_{\mathrm{G}}\right) \approx 6.5$ (see Figure 1) may point toward the existence of a slight enhancement in electron current, although it may also be due to spread in the data. It is felt that one can claim that no obvious enhancement in $\left(I_{+} / I_{+0}\right)$ and $\left(I_{\mathrm{e}} / I_{\mathrm{e} 0}\right)$ was definitely observed in this experiment.

Henderson and Samir (1967) presented results for the electron distribution in the wake of the Ariel I satellite at a distance of $\left(r / R_{\mathrm{A}}\right)=5$ downstream from the center of the satellite, where $R_{\mathrm{A}}=$ radius of the Ariel I satellite. The observations are for satellite perigee altitudes; i.e., 400 to $600 \mathrm{~km}$. A clear enhancement in the electron current was observed in the wake, although the enhancement did not rise above ambient (see Figure 3a). They also reported that a similar electron current enhancement was observed in the wake of the spherical ion probe that inadvertently served as a 


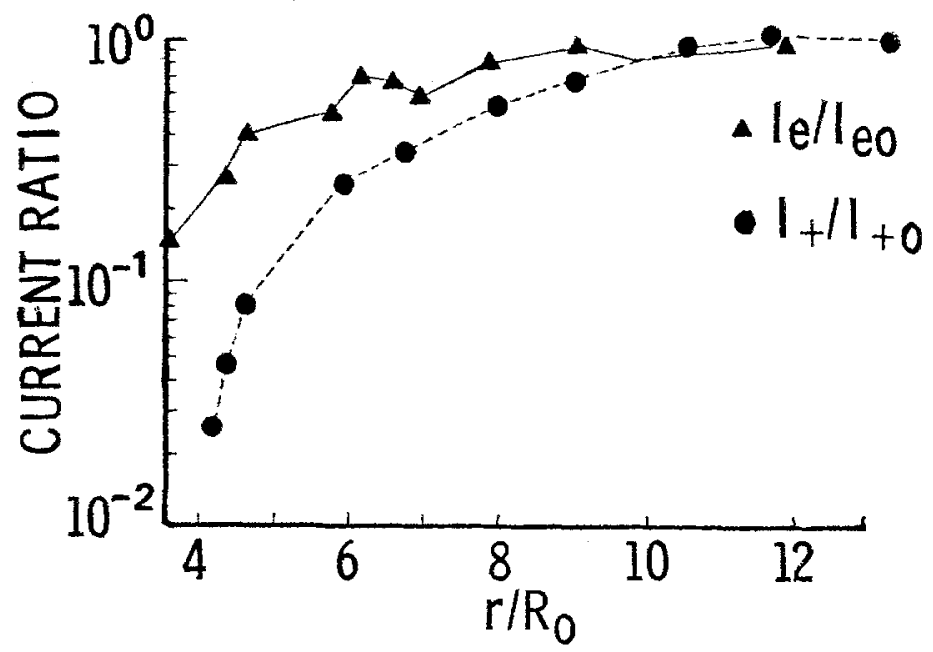

Fig. 1. Axial profiles of normalized electron and ion currents behind the Gemini 10 spacecraft, 20 July 1966 (after Troy et al., 1970). Altitude was $385 \mathrm{~km}$.

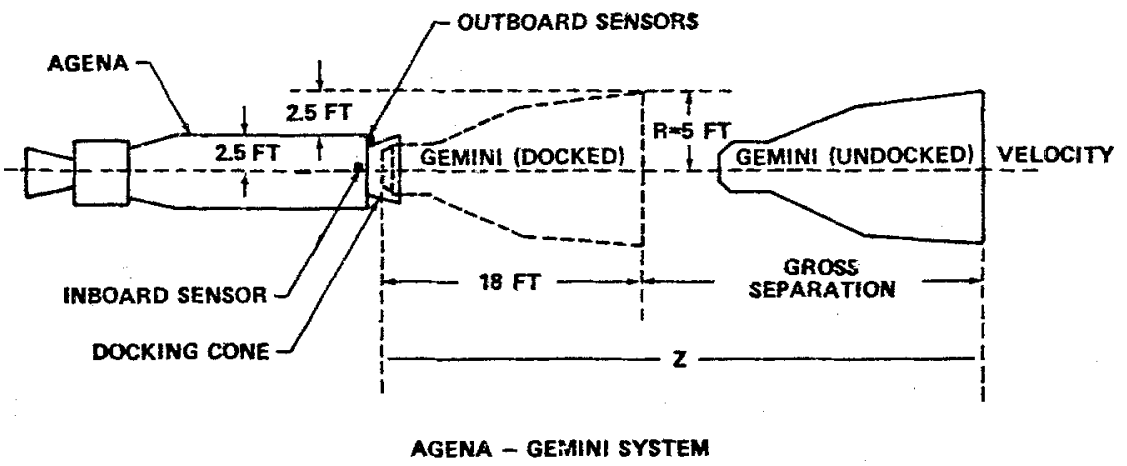

(a)

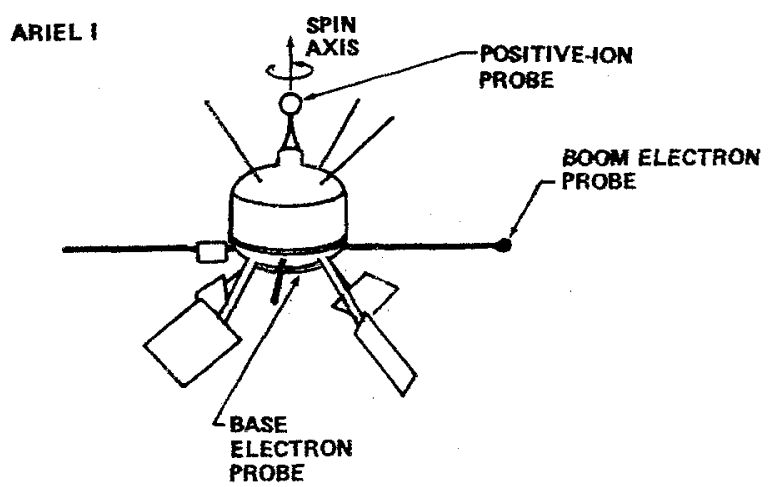

(b)

Fig. 2. (a) Gemini/Agena 10 docking/undocking flight configuration. (b) Ariel I configuration and probe location. 
- ONE MEASUREMENT

- MORE THAN ONE MEASUREMENT

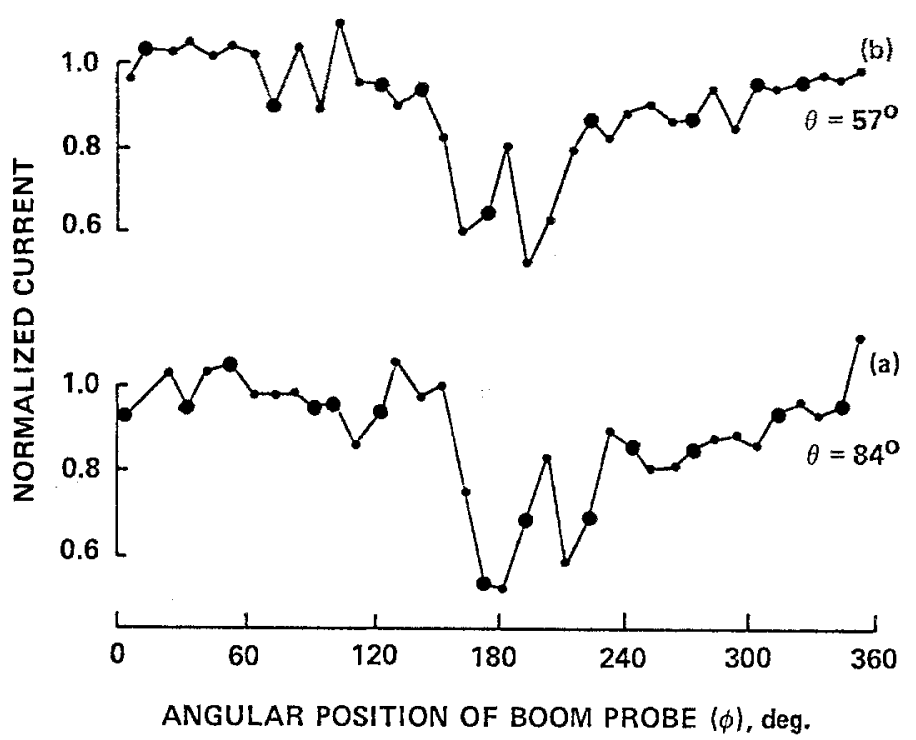

Fig. 3. Normalized electron current vs angular position obtained by the boom probe averaged over $10^{\circ}$ rotation for cases where the probes passed behind (a) the main body of the Ariel I satellite and (b) the spherical ion probe (after Henderson and Samir, 1967).

'wake-creating body' (see Figures $2 \mathrm{~b}$ and $3 \mathrm{~b}$ ). The ion probe was biased $6 \mathrm{~V}$ negative with respect to the main satellite body and was of radius $R_{\mathrm{p}} \approx(1 / 6) R_{\mathrm{A}}$, where $R_{\mathrm{A}}=$ satellite radius and $R_{\mathrm{p}}=$ ion probe radius. The body of the Ariel I satellite was at a potential of about $-1 \mathrm{~V}$ with respect to the ambient plasma.

Therefore, an apparent discrepancy seems to exist between the Ariel I results and the results from the Gemini/Agena 10 experiment where no obvious current enhancement was found. It should be mentioned that the results from the Ariel I were obtained by a guarded, planar electron disc probe mounted on a boom, while the Gemini/Agena 10 measurements were performed by planar R.P.A. type probes mounted on the surface of the Agena spacecraft docking cone. Also, the $\left(R_{\mathrm{G}} / \lambda_{\mathrm{D}}\right)$ ratio for the Gemini/Agena experiment was $\approx 10^{2}$, while the corresponding ratio for the Ariel I satellite $\left(R_{\mathrm{A}} / \lambda_{\mathrm{D}}\right)$ was $\approx 10$. The other space plasma properties were approximately similar.

Two possibilities exist regarding the discrepancy between the above in-situ observations: (1) no current enhancement existed behind the Gemini 10 spacecraft, and, since all parameters were essentially similar to the Ariel I parameters with the exception of the $\left(R_{0} / \lambda_{\mathrm{D}}\right)$ ratio, there exists a fundamental difference in the wake structures associated with 'large' (i.e., $R_{\mathrm{O}} / \lambda_{\mathrm{D}}>10^{2}$ ) bodies; or (2) a current enhancement did in fact exist behind the Gemini 10 spacecraft, but, for some reason, went undetected. 
A large number of laboratory simulation experiments have been reported where the characteristics of rarefied plasma streams over target bodies have been studied and can provide additional insight into the above question. Among the recent studies relevant in some aspect to the interaction between a spacecraft and its environmental space plasma we quote: Hester and Sonin (1969b, 1970), Sajben and Blumenthal (1970), Stone et al. (1972), and Fournier and Pigache (1972). The above studies were performed over a wide range of plasma flow parameters some of which are of direct interest for ionospheric satellites; i.e., $0.8 \leqslant\left(R_{0} / \lambda_{\mathrm{D}}\right) \leqslant 46 ; 5 \leqslant S\left[=V_{\text {flow }} / V_{\mathrm{T}}\right] \leqslant 24$; $0 \leqslant\left|e \phi_{\mathrm{S}} / k T_{\mathrm{e}}\right| \leqslant 10$ where $R_{0}=$ body radius, $V_{\mathrm{T}}=\left(2 k T_{\mathrm{e}} / m_{+}\right)^{1 / 2}$, and $\phi_{\mathrm{S}}=$ body potential with respect to the ambient plasma. All of the above studies indicate the existence of an ion current enhancement in the wake with a maximum amplitude at some point on the axis.

Figure 4 shows the relationship between the Mach number $(S)$ and the normalized distance $\left(r / R_{0}\right)$ at which the maximum ion current enhancement was observed for spherical bodies over a wide range of plasma parameters in the above studies. This not only attests to a dependence of the location of maximum current enhancement

LOCATION OF ION CURRENT PEAK (MAX. AMPLITUDE) VERSUS VELOCITY NUMBER

FOR SPHERICAL BODIES

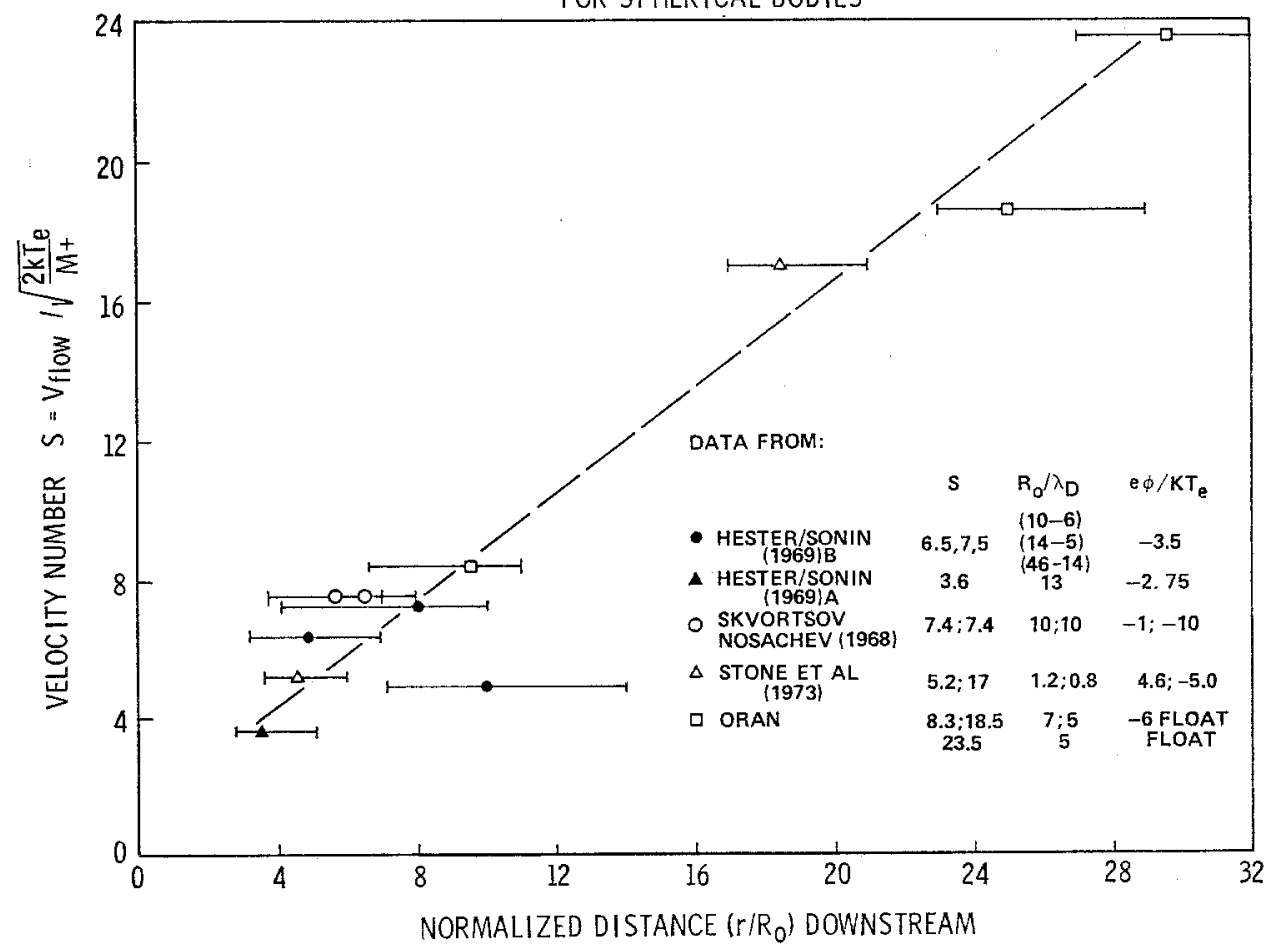

Fig. 4. The location of the point of maximum current enhancement on the wake axis vs the Mach number. 
on the Mach number, but, more fundamentally, to the fact that an ion current enhancement does exist in all cases for spherical bodies. The precise nature of the enhancement and the location of the maximum amplitude depend on the plasma parameters and to some extent on the cross-sectional geometry of the body exposed to the streaming plasma (see Oran et al., 1974a).

This structure and the dependence on Mach number can be explained by the actions on the free streaming ions of the self-consistent electric field existing in the sheath of the target body. The ions, as a result of this attractive force, are focused onto the wake axis; the amount of focusing depends on their directional kinetic energy (and, hence, $S$ ) and the field strength. Several theoretical models have predicted an axial ion current enhancement of this type downstream from bodies with circular cross sections exposed to the plasma flow (e.g., Call, 1969; Gurevich et al., 1969). The above effect is often described in the literature in terms of a 'two-stream' flow, referring to the conceptually simplified picture one obtains when considering the pattern on a plane containing the wake axis, or the wake created in a two-dimensional problem.

There is general agreement, then, between the various laboratory studies and theoretical models, quoted above, and the Ariel I results regarding the existence of a current enhancement at a distance of about $S \cdot R_{0}$ downstream. It should be noted, however, that while the Ariel I data provide evidence for the existence of a current enhancement, it cannot provide information on the precise location of the maximum enhancement, since the observations were made at a fixed radial distance from the spacecraft. In addition, the electron current enhancement observed at a distance of $5 R_{\text {Ariel }} \gg S \cdot R_{\text {Probe }}$ downstream from the spherical ion probe is in accordance with the results of several laboratory studies (e.g., Hester and Sonin, 1970; Stone et al., 1973) that show the ion current enhancement to persist downstream to distances $\gg S \cdot R_{0}$.

We should point out that the above-quoted laboratory studies apply primarily to ion fluxes, while the Ariel I measurements consist only of electron currents. However, in recent laboratory work by Oran et al. (1974b), both electron and ion current enhancements were observed on the wake axis. The enhancements in electron current found on the wake axis begin at distances $<S \cdot R_{0}$ and extend beyond this location axially. In no case did this enhancement exceed the ambient one. Based on the above laboratory and in-situ observations, one can expect both electron and ion current enhancements to occur in the wake of spacecraft unless there are additional mechanisms which can eliminate the enhancement.

Recently, Fournier and Pigache (1972) attempted a simulation of the transverse ion temperature in the O.N.E.R.A. wind-tunnel facility. It should be mentioned that the transverse ion temperature which they simulated is applicable as a nonMaxwellian transverse ion-velocity distribution. These experiments indicate that a non-vanishing ion temperature may smooth out peaks in the wake, which is in accord with the results of Taylor (1967). The peak enhancement we discussed may perhaps be influenced by that component. However, the Ariel I results which naturally 
include the ion thermal motion, are in accordance with the previously discussed laboratory studies which indicate the existence of an enhancement. These experiments did not simulate the transverse component of the ion thermal motion. This may have a bearing on the degree of applicability of theories which assume a 'cold ion' plasma. However, a definite quantitative conclusion as to the importance of the thermal component of the ion motion at different locations on the wake axis requires further parametric study. We should state again that the plasma flow parameters in the environments of both the Ariel I and the Gemini/Agena 10 were similar, the spacecraft potential $\left(\phi_{\mathrm{S}}\right)$ was $0 \leqslant\left|\phi_{\mathrm{S}}\right| \leqslant 1$ for both spacecraft, and the $\left(R_{0} / \lambda_{\mathrm{D}}\right)$ ratios differed by an order of magnitude.

If the difference in the $\left(R_{0} / \lambda_{\mathrm{D}}\right)$ ratio is, indeed, the reason for the non-existence of the current peak in the wake of a 'large' spacecraft, then we have gained an insight into the applicability of the 'two-stream' model for describing wake structure enhancements behind spacecraft. This result (if true) could apply to the interpretation of wake and sheath experiments to be performed on future Shuttle missions.

If, on the other hand, such an enhancement did exist in the Gemini 10 wake, the question remains as to why it was not observed. Recent studies performed in the plasma wind-tunnel facilities of the Marshall Space Flight Center showed the transverse width of the region of the enhanced ion current in the wake to be narrower than or of the order of the diameter of the target body (see Figure 5a).

From Figure 5b-c, which shows axial profiles of normalized ion current on the wake axis and $(1 / 2) R_{0}$ off axis, it follows that a misalignment of several Gemini radii or even (1-2) $R_{\mathrm{G}}$ for the Agena with respect to the Gemini wake axis could suffice for an existing peak not to be detected. In fact, misalignments of the order of $(1-2) R_{\mathrm{G}}$ in the Gemini/Agena 10 experiment were known to exist. Furthermore, if a current enhancement existed but its amplitude lay below ambient, as it does in the case of the Ariel I satellite, it may not have shown up clearly even for a profile on the wake axis.

In summary: three factors are involved in an attempt to explain the unobserved current enhancement on the wake axis of the Gemini spacecraft: (1) sufficient ion thermal motion existed to completely suppress such an enhancement, (2) large spacecraft (i.e., $T_{0} / \lambda_{\mathrm{D}}>10^{2}$ ) are not subject to a 'two-stream' behavior, and (3) an enhancement existed but was not observed. We submit that the Ariel I results together with the laboratory results do not support possibility (1). No theoretical or experimental work is available, at present, for $R_{0} / \lambda_{\mathrm{D}}>10^{2}$ that can either support or contradict a 'two-stream' behavior for large bodies. Therefore, until further studies are performed, no conclusion can be derived based on possibility (2). We suggest a possible experimental cause which can result in an existing enhancement not being observed.

The present re-examination of the available in-situ data in conjunction with laboratory and theoretical studies should point toward the experimental approach to be adopted in future space experiments in this area in order to remove such ambiguities. The discussion in this paper may also have relevance to the solar wind flow over 

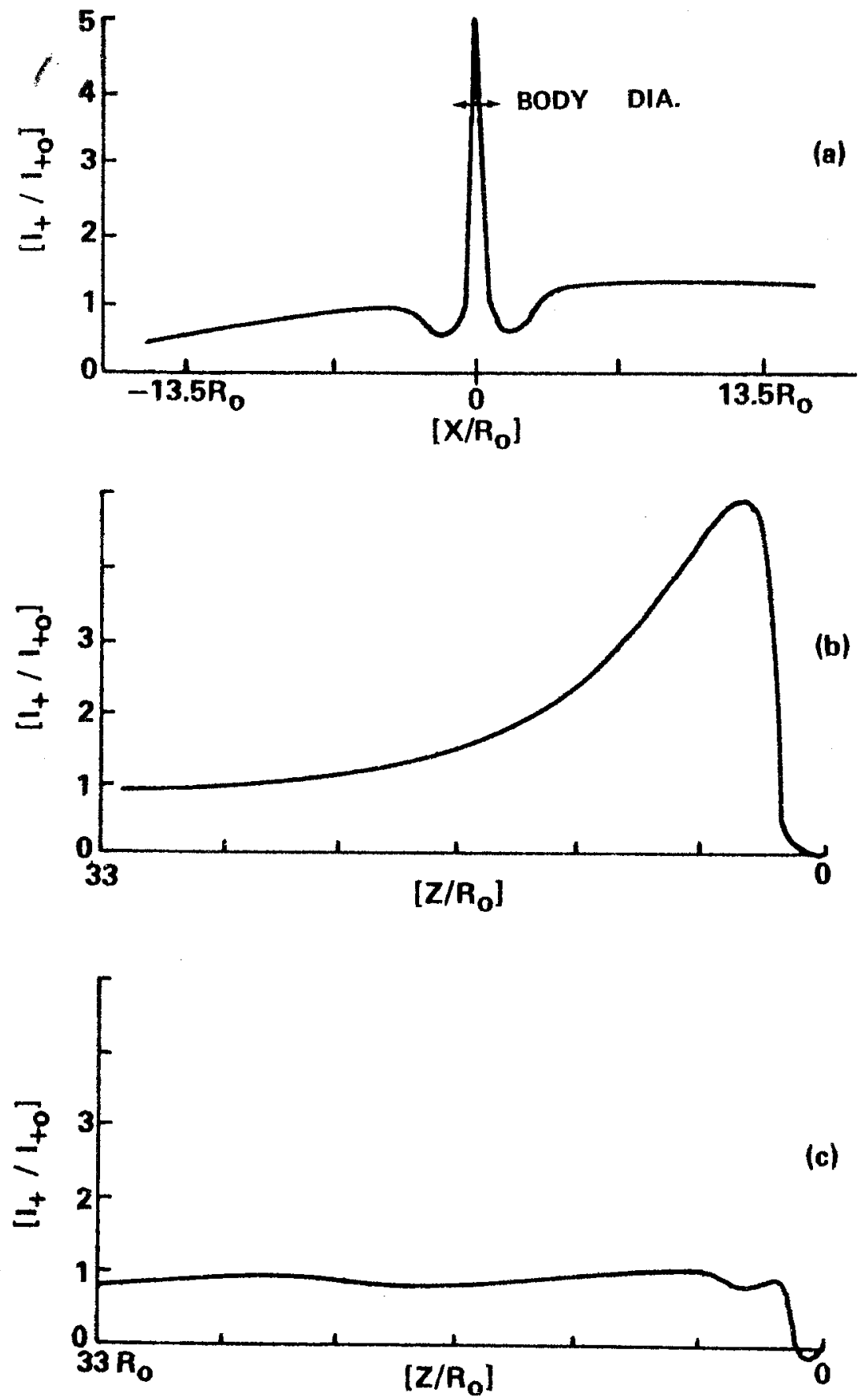

Fig. 5. Profiles of normalized ion current behind a sphere in a laboratory plasma stream with parameters: $S=11.6, R_{0} / \lambda_{\mathrm{D}} \approx 15, \phi_{\mathrm{N}} \approx-8$. (a) Transverse profile at the point of maximum enhancement; (b) axial profile centered on the wake axis; (c) axial profile $(1 / 2) R_{0}$ off the wake axis. 
non-magnetized bodies such as small asteroids and moons of planets where $R_{0} / \lambda_{\mathrm{D}} \gtrsim$ 100.

\section{References}

Call, S. M.: 1969, Plasma Lab. Report No. 46, School of Eng. and App. Sci., Columbia University, New York, N.Y.

Fournier, G. and Pigache, D.: 1972, J. Appl. Phys. 43, 454.

Gurevich, A. V. et al.: 1969, Space Sci. Rev. 9, 805.

Henderson, C. L. and Samir, U.: 1967, Planet. Space Sci. 15, 1499.

Hester, S. D. and Sonin, A. A.: 1969a, Rarefied Gas Dynamics, Sixth International Symposium, 1659.

Hester, S. D. and Sonin, A. A.: 1969b, AIAA Paper No. 69-673.

Hester, S. D. and Sonin, A. A.: 1970, Phys. Fluids 13, 641.

Oran, W. A. et al.: 1974a, J. Geophys. Res., submitted for publication.

Oran, W. A. et al.: 1974b, Planetary Space Sci., submitted for publication.

Sajben, M. and Blumenthal, D. L.: 1970, AIAA Paper No. 69-79.

Skvortsov, V. V. and Nosachev, L. V.: 1968, Kosmicheskie Issledovaniya 6, 228 (UDC 533.9.07).

Stone, N. H, et al.: 1972, Planetary Space Sci. 20, 1787.

Stone, N. H. et al.: 1973, JATP 36, 253.

Taylor, J. C.: 1967, Planetary Space Sei. 15, 155 and 463.

Troy, B. E., Medved, D. B., and Samir, U.: 1970, J. Astronautical Sci. 18, 173. 